\title{
Shaping the Discourse of Worker Health in the UK Construction
}

\section{Industry}

The UK construction industry is in poor health, statistics show rates of occupational illness significantly higher than for workers in any other industry. Recently, the scope of occupational health management on large sites has increased as public health has become included within the organisational remit. Concerns have been raised around the consequences of this development, its close relationships to Corporate Social Responsibility, and how this is now shaping the wider discourse. To explore this phenomenon, a critical discourse analysis of UK 'construction worker health' has been carried out, using the publicfacing data of ten large UK contractors. Findings show that 'public' has now overtaken 'occupational', the latter restricted to legal compliance presented as corporate citizenship, the former championed as evidence of benevolent organisational values. Yet public health concerns are limited to those of lifestyle and individual responsibility, whilst more complex issues around the social determinants of health as associated with work are missing from the discourse, separating organisations from the impacts of their work on their workers. A contemporary mapping of 'construction worker health' is developed for consideration by industry and academics to support future health initiatives and research.

Keywords: Corporate Social Responsibility, Construction Worker, Critical Discourse Analysis, Occupational health, Public health 


\section{Introduction}

Despite their seemingly unbreakable amalgam, health and safety are theoretically and practically very different things. The immediacy and impact of an accident has led to a prioritisation of safety in both practice and research, whilst health has been more neglected (Skan 2015) due to its 'slow-burn', and indeed the fact that it can be much more problematic to manage in practice. In recent years this inequality has become more apparent and to redress the balance health has been gaining priority within the UK construction industry, as demonstrated by its high profile on the London 2012 Olympic Park construction project (Tyers and Hicks 2012) with its adopted slogan of 'health like safety' (Waite 2012).

Occupational health in UK construction has also been influenced by the growing trend of governments to 'partner' with industry to achieve improvements in the public sector. One such example was the previous UK Coalition Government's Public Health Responsibility Deal (PHRD) launched in spring 2011, aiming to improve public health through ' ... a more collaborative approach to tackling the challenges caused by our lifestyle choices.' (Department of Health (DoH) 2015), with a specific Pledge (H10) for Construction and Civil Engineering Industries launched in 2013. Although currently in a hiatus, as the present Conservative Government have to date made no further development of this programme since they came to power in May 2015, theoretical challenges of this H10 Pledge, and its potential impact on the UK construction industry health management agenda, have been explored elsewhere (Sherratt 2015a; Sherratt 2015b). Issues of worker autonomy and personal freedoms were highlighted as potential concerns, analyses also illuminating the 
growing influence of Corporate Social Responsibility (CSR) on construction health in terms of its packaging and presentation (Rawlinson and Farrell 2010).

There are well-recognised links and overlaps between occupational and public health, not least the influence of work within the social determinants of health, the reasons why people drink too much, eat too much, smoke or use drugs (Wilkinson and Marmot 2003; Dollard and Neser 2013; Dhesi 2014), although such nuanced relationships often remain hidden. Instead, research often focuses on occupational aspects which then diffuse into public health concerns, although this connectivity is not always so clearly stated, and has often been explored through isolated trades or job roles (see Boschman et al 2011 for a study focused on bricklayers and construction supervisors), the influence of epidemiology evident in such specificity. Yet such focused research should arguably be supported by more holistic explorations that seek to examine construction industry health in a way which acknowledges both occupational and public health and the areas in between, and from a variety of perspectives.

This paper aims to make such a contribution through the examination of a specific discourse of UK construction industry health: the way large UK contractors' position worker health within their public-facing organisational identities. Such an approach does not seek to reveal actual industry practices, instead this analysis aims to reveal the presented relationships between public and occupational health and their relative prioritisations, as influenced by demonstrable CSR. This is a highly relevant discourse for construction worker health, as it is inevitably shaped by the intervention of CSR as 'propaganda' (Bernays [1928] 2005), which in turn dictates our societal expectations, the questions we should ask of organisations, and the parameters by which we then judge any aspect of life thus presented 
(Herman and Chomsky 1988); in this instance what 'good' worker health management 'should' look like for the UK construction industry. There is therefore the potential for the medium to, in part, start to determine the message (McLuhan and Fiore 1967), with consequences for practice within organisations and the expectations of clients seeking to further their own CSR when they engage with industry. It is hoped that this paper will in part provide something of a critical foundation for further research around worker health, with the potential to make a contribution to the development of effective improvements and initiatives in practice.

\section{Context}

\section{Occupational Health}

The UK's Health and Safety Executive (HSE 2015a) have reported that annually around 69,000 construction workers suffer from an illness they believe was caused or made worse by their work, a rate of illness statistically significantly higher than for workers in any other industry. Although the diversity of occupations in construction means not all workers are exposed to the same health hazards and not all occupations require the same physical capacity (Boschman et al 2011), specific health issues can be generically associated with construction work; for example incidents of work-related musculoskeletal disorders and lung problems are again significantly higher than in other industries. In terms of its practical management on sites, occupational health should be subjected to the same approach as safety; through robust management systems and the risk assessment process as required by the Management of Health and Safety at Work Regulations 1999. Industry is also supported by various initiatives, most prominently Constructing Better Health (2015a), which provides guidance, training and links to accredited occupational health providers. 
However, research suggests that occupational health within construction can be misunderstood and its management rendered relatively ineffective. For example, Thompson and Ellis (2011) found that health is often managed alongside safety as one coherent unit, rather than as separate aspects which require different approaches in their mitigation and minimisation. This has the potential to limit effectiveness should controls be applied from the perspectives of immediacy as necessary for safety, rather than the longterm view required for health. This was found by researchers on the 2012 Olympic Park; whilst the level of personal protective equipment (PPE) on site was good, '...access to equipment or procedures designed specifically to control [occupational health] risks (e.g. checks on noise levels ... well-maintained dust extraction equipment and use of antivibration handles) was less common' (Tyers and Hicks 2012:8). Whilst PPE is the most immediate, readily available and cheapest response to the identification of health risks within a construction process, it should only be considered the last resort from both a risk assessment and a long-term health management perspective (HSE 2015b).

Although efforts are certainly being made to improve the occupational health of the UK construction industry, and recent statistics do show evidence of such improvements (HSE 2015a), its management is arguably still in its infancy when considered alongside the myriad of systems, controls, work processes and practices that are now in place to manage safety on sites.

\section{Blurred boundaries: Occupational and public health}

The construction industry is perhaps more closely aligned with public health than some others; it needs 'healthy' workers for production - to walk and climb, to lift and move, to balance and level, to force and fit. There remains a heavy reliance on manual labour and 
skills within traditional work processes, and therefore health becomes a necessary characteristic of the construction worker, the big, strong 'beefy builder' stereotype (Sherratt 2015a). Yet, the UK construction industry loses on average 1.2 million working days through work-related ill health each year (HSE 2015c), to the detriment of productivity and output and, much more importantly, to the detriment of the workers themselves.

Economically, public health can be seen as a corporate concern; employers interested in mitigating economic losses suffered as a result of illness, and so the idea to manage public health alongside occupational health is often deemed an eminently practical and sensible approach (Healey and Walker 2009). Yet public health is also grounded in what are termed wider health inequalities, themselves described as 'wicked problems' (Dhesi 2014:30), where the complex interplay of factors such as status, social class, power, earnings, education and living standards all contribute to poorer or better health (Marmot 2004). Work does have a significant role to play here, and one of the policy objectives put forward by Marmot et al (2010:9) in 'Fair Society Healthy Lives' for the UK Government was to 'create fair employment and good work for all'.

Much has been made of this within contemporary society. Labelled the 'changing work environment' (Papadopoulos et al 2010), we now are becoming more aware and accepting of increased work intensity, long working hours, weekend work, increased percentage of employment under subcontractors, job insecurity and temporary or 'zero-hours' contracts. However, all of these aspects can negatively impact on worker heath, for example it has been determined that job insecurity actually poses as comparable a threat to health as having no job at all (Kim and von dem Knesebeck 2015). 
But for the UK construction industry, this is not a 'changing work environment' - this simply is our work environment. Hours on UK construction sites are often excessively long, the process of competitive tendering for winning projects creates an unstable work environment reliant on subcontracting, long supply chains, and a transient and fragmented workforce, all managed through bonus and payment schemes that encourage intensive work practices to support the constant demand for progress. For the construction workforce, so easily described as temporary and transient, never able to look too far beyond the next job or project, the potential for employment uncertainty to negatively influence worker health is clear. Taken together this creates multiple, often cumulative stressors within construction work (Beswick et al 2007; Cunradi et al 2009); fundamentally, it can be argued that the contemporary UK construction industry is structured to the inevitable detriment of construction worker health. And this is before other, perhaps more visibly unfavourable aspects of construction work are also considered, including, for example, the need to be responsible for the safety of others, working in dangerous conditions (Beswick et al 2007), managing rapidly changing tasks, high physical demands, cost pressures, the need to quickly make difficult decisions without sufficient information, low job control and the low levels of social support frequently found on sites (Boschman et al 2011).

It is therefore unsurprising that stress within the construction industry has recently been highlighted as yet another health problem, but despite increasing levels of concern reported occupational stress within the construction industry remains significantly lower than that for all other industries in the UK (Health and Safety Executive 2015a). Such low reporting is perhaps a consequence of the complex relationships between work and stress, and the 'hidden' nature of many of these stressors, closely linked as they are to a resigned acceptance that this is 'the way we do things around here'. That they are also operating 
within a male-dominated, macho industry (Ankrah et al 2009) must also be recognised. One in which stigmas remain around stress and mental health in general, and where not feeling 'tough enough' for the job could easily be identified as a stressor in its own right.

\section{Public health: The symptoms}

Such negative influences on worker health can clearly impact mental wellbeing; adverse psychosocial environments can readily lead to stress related disorders such as depression and even manifest physically in occupational health symptoms such as musculoskeletal disorders (Boschman et al 2013). Yet these stressors also constitute significant threats for what can be much more clearly identified as public health (Kim and von dem Knesebeck 2015) through their influences as social determinants of worker health (Wilkinson and Marmot 2003; Dollard and Neser 2013). Research in this field has suggested that many of the high risk health behaviours that foster chronic disease, such as smoking, drinking, obesity and drug taking, are connected with the workplace (Healey and Walker 2009:47). For example, Papadopoulos et al 2010 found higher levels of alcohol consumption, smoking, drug use and obesity among temporary workers than permanent employees, suggesting that transient work is detrimental to health in more ways than one.

Yet whilst Papadopoulos et al's (2010) work focused on the changing work environment within all industries, more focused research also lends support to the argument that construction work has considerable negative impacts on its workers' health, beyond the more tangible hazards it demands they regularly face on sites. For example, research in the US (Chin et al 2012) found that construction workers are almost twice as likely as the general working population to smoke, and they smoke more. Although this was also associated with sociodemographic characteristics such as age, male gender, lower levels of 
education and income, it was found to link to social pressures and stressful work, and higher exposure to occupational hazards. US construction workers have also been found to have the highest rates of heavy alcohol use when compared to other industries (Cunradi et al 2009), a finding mirrored by work carried out in Australia which also identified its construction workers to be at risk of hazardous alcohol consumption, and also illegal drug use (Biggs and Williamson 2012). Indeed, Minchin et al 2006 found that illegal substance use amongst any workforce is highest within the construction industry. In Holland, there is a prevalence of overweight construction workers with musculoskeletal disorders (Viester et al 2012), whilst in the UK, perhaps the use of the term 'Beefy Builders' within the PHRD press release was not too far from the truth, as on the 2012 Olympic park $41 \%$ of construction workers were overweight, $28 \%$ obese, and $30 \%$ had high blood pressure, a contributory factor to coronary heart disease (Waite 2012). More recently, the stark statistic has been published that more workers from construction trades committed suicide in the period 2010-2015 than any other profession in the UK (Construction Manager 2017).

\section{Construction Worker Health: Shaping the Discourse}

It can therefore be concluded that construction workers are not in the best of health. Poor occupational health can be directly linked to the hazards they face within the workplace such as dust, noise and the need for heavy manual labour. They are also vulnerable to poor mental health as occupational stressors such as dangerous work, production pressures, rapid and complex decision making and the responsibility for the safety of others are also prominent aspects of the working day. More fundamentally, they work within an industry structure that causes further psychosocial harm; transience, temporary work contracts, lack of job security and long working hours are recognised as equally damaging to worker health 
as dust and noise. And this has perhaps inevitably led to a workforce in which high levels of smoking, drinking, over-eating and drug use can be seen as symptoms of such cumulative workplace issues, readily able to provide 'cures' for these stressors, administered by the workers themselves.

This is perhaps a rather damning diagnosis, and one that has not gone unnoticed; worker health is now becoming as important as safety and the UK construction industry is making concerted efforts to tackle poor worker health, both occupational and public. However, given the complexities of the relationships as outlined above, caution and careful consideration of this wicked problem should perhaps be made before 'off-the-shelf' solutions are prescribed. These are issues and concerns that cannot be resolved with the simple application of PPE; they relate to much more fundamental aspects of the way the construction industry 'works' as a whole, and as a result are much more difficult to change. Unfortunately, health management within any occupational context is often limited to the superficial; indeed 'work-site wellness' programmes rarely include changes or improvements to fundamental working conditions, and efforts are instead directed to what can be more easily controlled such as behavioural factors and individual 'lifestyle' issues that can be used to deflect '... attention away from serious examining the effects of corporate cultures or the work environment' (Conrad 2005:546). Such superficiality can readily be found in the construction industry; analysis of Dutch construction worker health programmes identified a focus on worker lifestyle improvements (Groeneveld et al 2011), or on decreasing work demands through ergonomic measures (Oude Hengel et al 2012), whilst others suggest that such programmes are in practice only used by the already healthy part of the workforce (Viester et al 2012). Indeed, in the UK such problems have been 
recognised by the Health and Safety Executive (2016), who clearly state that organisations should 'manage risk, not lifestyles', referencing UK occupational health law, and although '... helping workers tackle lifestyle issues like smoking or diet may be beneficial ... it is not a substitute for this'.

Yet it is arguable that various initiatives, such as the now-defunct PHRD, support and even facilitate such superficiality (Sherratt 2015a), providing a distraction from the more complex problems of occupational health ingrained in construction industry operations by shifting attention from the workplace to the worker, from the underlying occupationally-triggered social determinants of health to the more simplistic 'public health' concerns of their symptoms, all the while allowing the relationship between the two to remain obscured. Indeed, the way construction health (and safety) has recently been subsumed under the remit of CSR within construction organisations (Rawlinson and Farrell 2010) has the potential to reinforce and perpetuate such practices. The very public face of CSR seeks to not only inform and advise how the construction industry and individual organisations are approaching and managing the problems of worker health, but it is also able to shape both worker and wider societal expectations of what this 'how' should look like. By its very nature, CSR prefers to address issues with ready metrics and photogenic output that can be easily commodified to provide 'content' and other evidence of the manifestation of CSR in practice, through organisational reports, social media presence and other public relations (PR) activities (Ennals 2011). CSR may struggle to resist the temptation to focus on the superficial symptoms of poor worker public health instead of problems associated with site management, work structure and organisation, but it is through this very process that the 
construction industry may itself be actively shaping the wider discourse around construction worker health into one of superficiality and worker personal responsibilities.

This should not go unexplored, or indeed unchallenged. Discourses in turn come to influence and shape both culture and practice (Gergen and Gergen 2003), and therefore it is perhaps timely to now examine this process in depth, to illuminate and examine the dominant discourses of construction worker health as they reveal or obfuscate the worrying reality of poor worker health. It is also timely that this should work should be undertaken before our shared understandings of construction worker health and its management become moulded or indeed ossified into the superficial shapes as prescribed by demonstrable CSR.

\section{Methodology}

In order to begin to empirically explore the discourse of UK construction worker health and how this is being shaped by CSR in practice, a critical discourse analysis (CDA) (Fairclough and Wodak, 1997) was carried out of construction industry public-facing documents that specifically reference worker health.

Grounded in a social constructionist epistemology (Burr, 2003; Gergen, 2009), CDA is not only able to illuminate and explore what is being constructed, but the process of this construction itself (Hacking, 1999). By exploring the discourses that make up our social worlds from acknowledged and explicitly critical perspectives, how they influence knowledge and beliefs, and how they are themselves shaped by ideology and power (Ness 2010) can be revealed. The approach seeks to unpack the way we position and create shared understandings of phenomena, examining the processes and functions of the discourse (Gergen, 2009) within situated contexts. A consequence of this epistemological 
position is that generalisation of these findings cannot be made from within traditional considerations (Lincoln and Guba 1985), and indeed CDA itself makes no claim to either truth or objectivity and is therefore inevitably grounded in one researcher's interpretation of the world. However, this does not devalue its potential contribution as CDA instead seeks to provide insight and illumination of a phenomenon in context, and so through its nuanced examinations and critical explication it is arguably a highly appropriate approach to explore the complex relationships between construction worker public health, occupational health and CSR in depth.

The sample for analysis comprised the ten construction contractors present on the UK's 'top ten' largest UK contractors as defined by 'yearly work won including civils September 2014 31 August 2015' (Building 2015). Such purposive sampling was used to ensure representation of the research phenomenon of interest (Payne and Payne 2004), rather than abstract statistical criteria; the size and success of these contractors reflecting a proactive approach to organisational health (and safety) alongside a mature development in terms of CSR activities. Although as previously noted no claim is made to industry-wide generalisability from this sample, it is suggested that the operational practices of other large UK contractors are likely to be similar, not least because they actually number relatively few in total; the vast majority of projects by value in the UK are constructed by a small number of organisations. As any industry is often led by its most successful companies, this sample is arguably highly appropriate given the influence these ten organisations will have on shaping the wider discourse through their individual CSR practices.

The data drew on two different public faces of these ten organisations - their corporate websites and their formal response and commitment to the PHRD Construction Pledge, as 
published on the UK government's now archived PHRD webpage. The use of web sites and web data as documentary sources can prove useful (Rawlinson and Farrell 2010), as they can be considered 'public documents of private origin' developed through the collective authorship of the organisation itself, and are therefore authentic, credible and representative data (Scott, 1990). A systematic approach to the websites was made, to ensure capture of all relevant pages, through direct links and a search for the keyword 'health'.

It must of course be acknowledged that both data sources are 'presented' data (Webb et al. 1966) designed to portray a positive image, yet this is of course highly relevant here. Indeed, the study can claim high levels of ecological validity given the aim of the research, there is arguably no more robust data source for work exploring the influence of CSR on a phenomenon than CSR itself. It is precisely the way health is positioned by these organisational identities through formal policies and displays of organisational commitment that is sought, in order to examine its contribution to the development of the wider discourse of construction worker health that will have 'social consequences ... such as influencing the social beliefs and actions of the recipients' (Van Dijk 2008:5) as well as setting wider societal expectations.

\section{Findings and discussion}

The positioning of health (and its seemingly inevitable partner, safety - subsequently noted as 'H\&S' where they occurred in this format) within the organisational webpage data could be traced through two distinct paths. The first travelled via 'sustainability', 'responsibility', or, more explicitly, 'corporate responsibility', to a page in which H\&S was a link alongside those leading to environment, governance and community. The second positioned H\&S 
within some form of corporate identity; 'about us', 'who we are', and 'how we do it', the H\&S link here sitting alongside those leading to values, culture, strategy and history. These two different approaches were split equally between the ten organisations, five adopting a 'sustainability' approach, and five an 'identity' approach. Interestingly this reflects one of the wider debates around CSR; is it something the company does, or something the company is?

From this perspective, health, occupational or public, becomes either a practice or a 'value', and therefore its management and consideration can become very different things, although further analysis did not reveal any coherence within the subsequent discourses of health as located beyond these two pathways. All but one of the sample organisations had previously 'signed up' to the PHRD, and all but one had published an 'Action Plan' and subsequent 'Annual Update'. Within this data, health was explored with more focus, as would be expected, however when considered alongside the corporate data, several nuanced facets became identifiable within the wider discourse of health that emerged.

\section{Unbreakable Amalgams}

The fact that 'H\&S' rapidly became the shorthand during the analysis of the website data was reflective of the fact that safety is still very much the dominant partner within the H\&S amalgam. Health was often negated for the maximisation of safety; where H\&S formed the link or page title, the content often developed through explication of safety leadership, ways of working safely, safe behaviour, safety incidents and Accident Frequency Rates (AFRs).

There was also a 'muddling' of health with safety within the discourse, as well as within its management in practice. For example, one page stated that 'poor health in the workplace 
can present significant safety risks'. Although healthy workers are needed for certain jobroles, such as crane drivers, here health is positioned only as a predetermining factor of safe practice, rather than an occupational consideration in its own right. Another stated that 'employees with safety critical roles for example those at high risk of hearing damage, vibrations etc. are given regular health screenings'. Again, occupational health issues are aligned with safe working and management in practice, and, worryingly, the health intervention is positioned during and after the event as an acceptable approach carried out for safety reasons alone, rather than any proactive prevention of poor occupational health. Yet this is itself muddled as health screening involves the examination of workers to look for conditions that can be best treated early, such as various cancers, in this scenario health surveillance is actually required to monitor the workers hearing throughout the role, the considerations of health lacking in their relation to good practice.

This was also identifiable in the PHRD data, where it was noted that checks would be carried out to ensure that 'safety critical workers were fit for work', again the emphasis placed on safety, health only associated with the workers ability to carry out their allocated tasks correctly rather than any wider occupational considerations. Where such 'muddling' occurred, health was often the losing party, safety dominating both the organisational discourse and any recourse to practice.

Within the PHRD data a new amalgam was created, that of 'health and wellbeing'. The emergence of this relationship remains unclear, with any definition or explication of 'wellbeing' lacking within the data. Within this partnership, health was more dominant, although this relationship could not be as easily defined as for ' $\mathrm{H} \& \mathrm{~S}^{\prime}$, and health was also associated with lifestyle, as 'healthy lifestyles' or 'healthier lifestyle choices', creating closer 
and perhaps more recognisable associations with wellbeing than to safety. Within the PHRD data, 'health and wellbeing' was used as something of a discursive shorthand to encompass all that was not safety within this particular context, very much reflective of how H\&S was mobilised within the organisational website data, although with different consequences in terms of overall focus.

\section{Healthy Work}

Despite its relevance to construction work, and the industry's poor record in this area, occupational health was actually very limited in its recognition within the corporate website data. Although there was acknowledgement of 'ill health caused by work', this was not a dominant positioning of health within the wider discourse, and its contribution was limited to associations with management, and most specifically the risk assessment process.

For example, one organisation stated that 'all our businesses will conduct health checks and health risk assessments to ensure there is no long-term harm to health from working in our business.' Yet this positioning of risk assessments as pro-active efforts, rather than the minimum legal standards they are for any construction work task (HSE 2015d), is a highly misleading construct within the wider discourse of health, although one that is commonly employed (Sherratt 2015a). Seeking to create an enhanced positive image around a legally required activity is suggestive of PR 'spin' and the need to position organisational efforts as 'above and beyond' minimum standards in the desire to demonstrate corporate citizenship. Within this wider context, it could therefore be considered unsurprising that this dataset did not reveal more details of occupational health management in practice, such mundanity unable to make a strong contribution to what is essentially marketing literature. But this argument can itself be challenged by the fact that this was certainly not the case for health's 
long term partner, safety. The discourse of safety management within the dataset constantly sought to go beyond mere legal requirements; the development of safety programmes, zero targets, site management practices and training were all championed as evidence of organisational commitment to safety. Yet occupational health did not receive this same consideration, either proportionally in terms of content, or in the level of detail accorded to its management in practice, and instead was simply reduced to the lowest common denominator of management; that of the minimum legal framework with which all must comply.

Within the PHRD data, occupational health was positioned more prominently and more practically, something previously identified through broader-brush analyses of this data set (Sherratt 2015b) but which remains surprising within this context, perhaps reflective of the difficulties of separating occupational from public health given their considerable potential for overlap. The need for compliance with legislation, including worker health surveillance was more explicitly considered, and occupational health was associated with practice; risk assessment and, most interestingly, specific aspects such as dust management, muskoskeletal disorders and respiratory problems were considered by several contractors to be areas of priority. The emergence of such mundane occupational practice within a discourse ostensibly around public health can be considered reassuring, suggestive of a prioritisation of the management of the most common occupational health risks found on sites, and which therefore surface quite naturally as industry priorities in any discourse of construction industry health.

Whilst the corporate websites are the formally designed public faces of the organisation, their PHRD contributions are much more hidden, although still readily available to both the 
public and prospective clients, should they know where to find them. Whether the authors are the same is certainly a point for consideration. The language, structure and presentation of the PHRD data was much less 'polished', suggesting a much more authentic industry voice. Indeed one organisation's PHRD Annual Update even begins with the statement that 'I think it is fair to say that all major contractors have fallen behind with the occupational health issues' (sic). It is therefore interesting to note the differences in the discourses of occupational health within these two contexts; the corporate webpages presenting straightforward compliance as evidence of over-and-above commitment whilst the PHRD data associates health much more with occupational practices and hazards than would perhaps have been expected.

\section{Healthy Lifestyles}

The more common manifestation of health identifiable within the webpage data, was as an area of 'concern', frequently linked with wellbeing, as something 'supported' by the organisation but without any corresponding level of detail or operational specificity. This approach, an example being the organisational intention of 'promoting health and wellbeing for everyone who works with us', avoids any description of occupational activity, and instead contributes to the development of a discourse focused more on public health. Although in some instances, both occupational and public health were explicitly considered within a simple, broad reference to 'health', public health issues were themselves much more dominant within the wider health discourse (as extracted from safety or even 'H\&S') found within the data. This was evidenced through explicit reference to the PHRD, companies stating their commitment to the Pledge, through the presentation of detailed health and wellbeing programmes seeking to educate and encourage lifestyle changes, or 
simply through core 'value' statements that 'we support health and wellbeing'. Although safety remains prioritised, public health has arguably superseded occupational health as the dominant health of the UK construction industry.

The PHRD data presented a different discourse around public health, one much more focused on action, as would be expected within the context of the 'Delivery Plan' being responded to. Here, 'engagement', 'awareness', 'change' and 'promotion' were associated with myriad initiatives, including events, campaigns, webinars, posters, factsheets and roadshows. Within the PHRD public health is something to be 'done', something active and tangible. This was also evidenced by the inclusion of programmes for health screening of the workforce as part of the Delivery Plans, closely associated with audits, data and metrics. Yet health screening is itself keenly debated within wider public health research, grounded as it is in various ethical and moral dilemmas (Sherratt 2015b). Whilst a 'Health MOT' for workers could include regular occupational health surveillance for any at-risk workers necessary to meet legislative criteria, it could also include various screening programmes seeking to test for other, non-occupational related conditions such as prostate cancer (popular in male dominated environments), and the addition of 'diet and lifestyle' programmes develops it into something even more potentially intrusive. Such programmes were also highlighted within the webpage data, although here the discourse was one of engagement with the workforce; offerings of on-site wellness screening and healthscreening clinics with the overall aim of 'building a healthier workforce' contrasting to the more clinical discourse of surveillance and control found within the more practical PHRD data. Within both data sets, the management and organisation of healthy worker lifestyles was positioned as a specialism, something beyond the capacity of the organisations themselves. The appointment of specialist consultants with various levels of accreditation 
and qualification to undertake both screening and interventions providing some measure of reassurance within these very pro-active and potentially invasive activities.

However, this activity and investment was not most prominently associated with occupational health, work stressors, nor the social determinants of health. Instead, a wide variety of public health 'problem issues' could be found within both datasets, including for example diabetes, high blood pressure and stress. But although the 'management of fatigue' and the need to 'support the reduction of ill health caused by stress' was noted within the PHRD data, there was no consideration of how to achieve the reduction or elimination of the causes of such stress or fatigue, they were simply to be treated through their symptoms. And as symptoms, they were closely associated with lifestyles, or more specifically 'lifestyle choices'. Indeed, the dominant discourse of health found within both the organisational webpages and the PHRD data was that of public health as lifestyle; the promotion of active lifestyles, smoking cessation, alcohol reduction and healthy eating most prominent within the data as a whole.

Yet these are issues that relate to individual choice and as such they are areas where notions of positive liberty and personal freedoms become highly significant (Sherratt 2015a). Indeed only one reference was found within the data to acknowledge the 'personal choice' of the worker, otherwise assumptions of 'good' health and 'the right choices' remained unarticulated and therefore unchallengeable (Sherratt 2015b). Yet these are also areas where the organisation is able to distance themselves from their manifestation within the workforce. These are individual issues, not organisational, clearly problems of public not occupational health, and so the organisation can confidently take on a benevolent position, helping the workforce make the 'right decisions' about their health and lifestyles, 
whilst the possibility that they have a significant role to play in the emergence of these health issues as a whole remains unremarked, even positively ignored. The atomisation of the workforce into individuals with responsibility for their own health is further reinforced by the inclusion of health screening processes, each worker reduced to a single point in the organisations database, readily able to be identified and 'supported' towards a predetermined better health. The wider discourse of public health found within both data sets is one of benevolence and engagement, yet does, as Conrad (2005) suggests, focus on the individual and their 'lifestyle' issues, and as such is able to deflect attention or enquiry away from any detrimental work practices within the organisations or industry as a whole.

\section{The Workforce}

The role the organisations gave themselves within this discourse was that of a provider of pastoral care, a supporter for the benefit of their workforce. But who this workforce actually is remains much more obscure. As noted above, reference was made within the webpage data to 'everyone who works with us', but in just one case was the supply chain specifically highlighted as a partner in the organisational public health improvement strategies. More frequently, the organisations 'workforce' remained obfuscated; 'all our people' providing a lack of specificity in the practical implementation of the health and wellbeing programmes. Indeed the difference in programme goals also adds to the intangibility of the discourse, the imagery of offices and computers used to illustrate the need for 'active lifestyles', something perhaps less relevant for those working on site than those based in the head office. More specificity was found within the PHRD data, in which the involvement, engagement and consultation of the supply chain was more clearly 
highlighted, but there was a relative lack of process and practice, when compared to other aspects of the Delivery Plans.

This lack of detail in terms of programme implementation is perhaps reflective of the inherent problems in maintaining a transient and fragmented workforce, which also includes large numbers of the self-employed. More cynically, the vagaries of this approach also enables organisations to make commitments to education, training and health screening that seem more generous than perhaps they are in practice. Whilst one organisation's webpage stated the aim of ' $75 \%$ of our employees [to be] using the programme by 2020 ', this will in fact be far less than the number of workers that will actually contribute to their construction outputs in practice.

\section{Image is Everything}

An interesting aspect of the data collection process for the webpage data was the location of much of the 'health' data beyond H\&S webpages. Formal policies could only be found for three of the ten organisations, the majority championed their health activities via the news or press pages of their websites, health commodified into PR content. Initiatives, activities and their associated prizes and awards were presented and supported by images of a healthy and happy workforce. This is perhaps unsurprising given the functionality of the webpage data, however the prominence of the same types of initiatives and events within the PHRD data, although presented far more prosaically, does lend support to the argument that such ventures do indeed have dual-purpose; to demonstrate organisational health activities, but also to do so in a way that is readily able to feed the ever-hungry PR machines. The provision of a few bowls of fruit to provide positive content is indeed far 
more visually stimulating than the provision of face fit dust masks; for one thing the latter inconveniently obscure the smiles of the workforce modelling them.

Yet, more seriously, it can be suggested that this 'packaging of health' as revealed by the data has indeed shaped the discourse, and influenced the emphasis on public health over occupational, a focus on the symptoms and not the causes, providing evidence of the medium shaping the message (McLuhan and Fiore 1967), and the wider discourse of health within construction.

\section{Conclusions}

Although focused on a very specific dataset, this research has been able to illuminate and present the discourse of construction worker health as presented through organisational CSR. With regards to overall prioritisation, health remains very much in second place to safety, but more surprising is that public health has surpassed occupational health as the dominant health of the wider discourse. Although the emergence of occupational concerns within the PHRD data, a space specifically set out for public health issues, goes some way to alleviate this, and indeed hints at an ongoing organisational considerations of practice which are to be commended, this is perhaps not sufficient to influence the discourse.

It is within the corporate webpages, the prominent public face of the construction organisations, that this increased focus on public health has in turn shaped health towards specific lifestyle symptoms and so created cause for concern. This is a discourse which places responsibility for health firmly onto the workers, enabling organisations to take on the role of benevolent guide, supporting workers as they make the 'right' choices about their lives, whilst ignoring the influence both organisational and industry-wide practices have in terms of work stressors and the social determinants of health. It is this latter fact 
should be much more clearly acknowledged within the debates around construction worker health, and organisational perspectives need to broaden beyond their 'beefy builder' workforce to recognise their own responsibilities and the consequences of their own actions in practice. The active influence of CSR in maintaining a focus on lifestyle, an area ripe for the winning of awards and production of photogenic and commodifiable content should also be challenged, before the medium does become the message.

Overall, this research has been able to reveal a discourse of worker health that is unlikely to support significant developments in occupational health management, shaped as it is by the influences of demonstrable CSR. But it is arguable that construction should be one of the UK's healthiest industries; workers are outside in the fresh air, they are mobile, they are able to use their muscles on a daily basis, to stretch and flex and tense. Indeed, a study of bricklayers in Holland found that their risk of mortality due to circulatory diseases was lower than average, a favourable effect of this trade for health (Boschman et al 2011). But construction workers are hampered by their poor occupational health, work stressors and their influences on the social determinants of health. The way the industry is organised, the way work and our workforce is structured, the way we do things round here, all of these hamper the health of construction workers, emerging as superficial symptoms of poor diets, drinking and smoking. To challenge these fundamental issues at their heart is much more critical than raising concerns about a lack of fruit.

In order to distil the contribution made by this research into something tangible, and present something of a roadmap to encourage both industry and academia to travel backwards from the symptoms to the causes, to explore and better understand how and why our industry is structured to the detriment of worker health, and what we must do to 
in Figure 1.

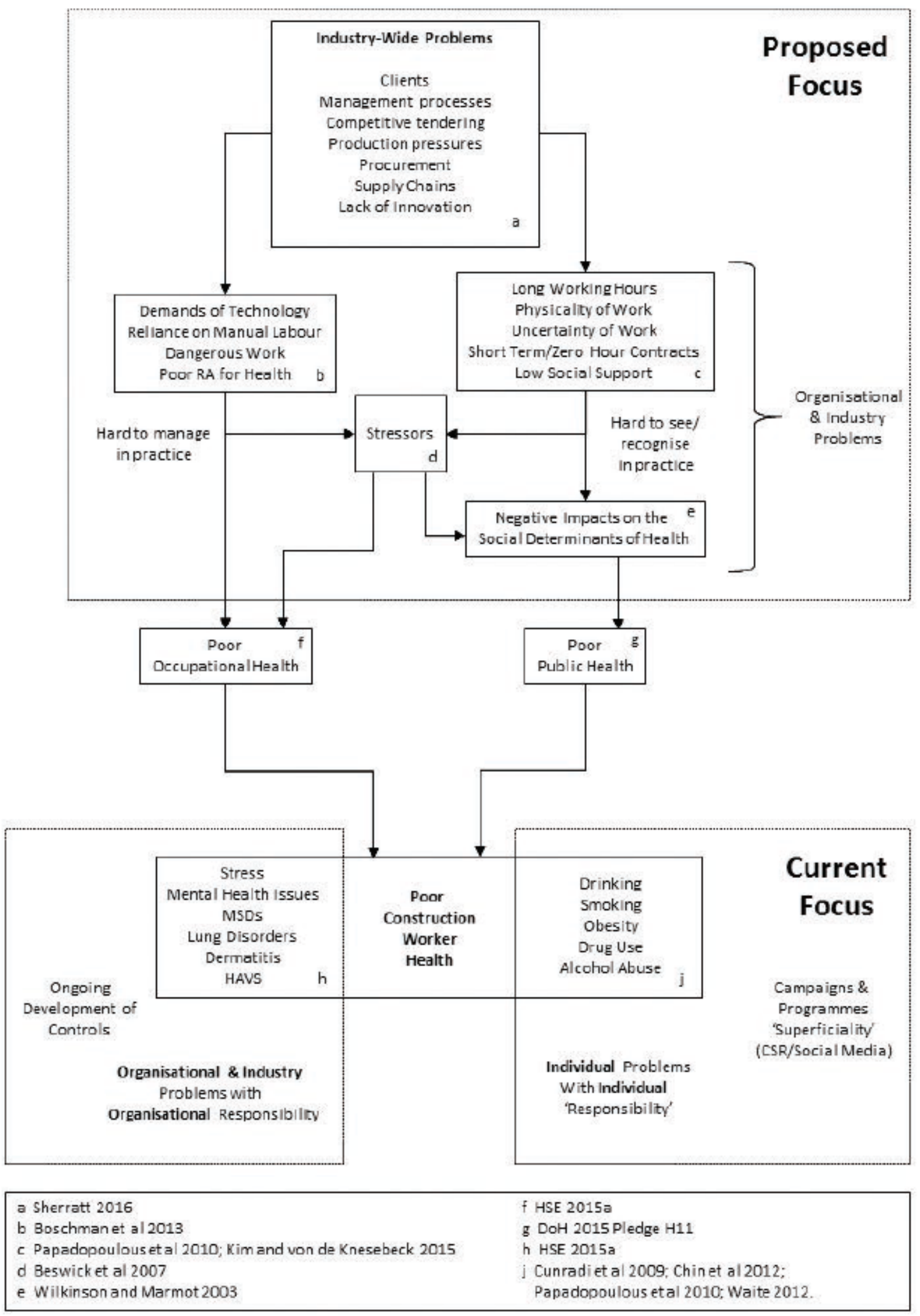


By bringing together the findings of this research and those from previous work undertaken both within and beyond the construction industry, a clearer picture of construction worker health emerges. The contribution of this research to the mapping is made through both its creation but also the illumination of the dominant discourse of construction worker health, and the way it is shaping contemporary practice to focus on the individual and their lifestyle choices, rather than the more unpalatable, complex and wicked causes of our industry's ill health. Further research is recommended to develop a strong evidence base to validate and verify these concerns specifically within the construction industry context, which will in turn be able to support the development of effective changes to work processes, structures and practices, with the goal of improving construction industry health overall.

\section{References}

Ankrah, N., Proverbs, D. and Debrah, Y., 2009. Factors influencing the culture of a construction project organisation. Engineering, Construction and Architectural Management. 16 (1), 2647.

Bernays, E., 1928 [2005]. Propaganda. New York: Ig Publishing.

Beswick, J., Rogers, K., Corbett, E., Binch, S. and Jackson, K., 2007. An Analysis of the Prevalence and Distribution of Stress in the Construction Industry. Norwich: Health and Safety Executive.

Biggs, H. and Williamson, A.R., 2012. Safety impacts of alcohol and other drugs in construction: development of an industry policy and cultural change management program. In: Smith, S.D (Ed.), Proceedings 28th Annual ARCOM Conference, 3-5 September 2012, Edinburgh, UK. Association of Researchers in Construction Management, 445-54. 
Boschman, J.S., van der Molen, H.F., Sluiter, J.K. and Frings-Dresen, M.H.W., 2013. Psychosocial work environment and mental health among construction workers. Applied Ergonomics 44, 748755.

Boschman, J.S., van der Molen, H.F., Sluiter, J.K., and Frings-Dresen, M.H.W., 2011. Occupational Demands and Health Effects for Bricklayers and Construction Supervisors: A Systematic Review. American Journal of Industrial Medicine, 54, 55-77.

Burr, V., 2003. Social Constructionism, 2nd Edn. Routledge, East Sussex.

Chin, D.L., Hong, O.S., Gillen, M., Bates, M.N. and Okechukwu, C.A., 2012. Heavy and Light/Moderate Smoking Among Building Trades Construction Workers. Public Health Nursing, 30(2), 128139.

Constructing Better Health, 2015. About CBH [online]. Constructing Better Health. Available from: http://bit.ly/1LcU6GI [Accessed 4th November 2015].

Construction Manager, 2017. More suicides in construction than any other industry, Construction Manager, 20 March.

Conrad, P., 2005. Wellness in the Work Place: Potentials and Pitfalls of Work-Site Health Promotion. In: P. Conrad (Ed) The Sociology of Health and IIlness: Critical Perspectives. 7th Edition. NYC, USA: Worth Publishers. 540-549.

Cunradi, C.B., Todd, M., Duke, M., and Ames, G., 2009. Problem Drinking, Unemployment, and Intimate Partner Violence among a Sample of Construction Industry Workers and their Partners, Journal of Family Violence, 24, 63-74.

Department of Health, 2015a. About the Public Health Responsibility Deal [online]. UK Government.

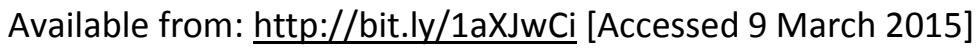

Dhesi, S., 2014. The role of health and wellbeing boards. In: A. Knight, V. La Placa and A. McNaught. (Eds) Wellbeing, Policy and Practice. Banbury: Lantern Publishing Limited. 27-36. 
Dollard, M.F. and Neser, D.Y., 2013. Worker health is good for the economy: Union density and psychosocial safety climate as determinants of country differences in worker health and productivity in 31 European countries. Social Science and Medicine, 92, 114-123.

Ennals, R., 2011. Labour Issues and Corporate Social Responsibility. In: S.O. Idowu and C. Louche (Eds) Theory and Practice of Corporate Social Responsibility. London: Springer. 143-158.

Fairclough, N. and Wodak, R., 1997. Critical Discourse Analysis. In: T.A. van Dijk (Ed) Discourse as Social Interaction. London: Sage Publications Limited. 258-284.

Gergen, K.J., 2009. An Invitation to Social Construction. 2nd Edition. London: Sage Publications Limited.

Gergen, M. and Gergen, K.J., 2003. Social Construction: A Reader. London: Sage Publications Limited.

Groeneveld, I.F., Proper, K.I., van der Beek, A.J., Hildebrandt, V.H. and van Mechelen, W., 2011. Short and long term effects of a lifestyle intervention for construction workers at risk for cardiovascular disease: a randomised controlled trial. BMC Public Health 11, 836.

Hacking, I., 1999. The Social Construction of What? London: Harvard University Press.

Health and Safety Executive, 2015a. Health and safety in construction sector in Great Britain 2014/15 [online]. Health and Safety Executive. Available from: http://bit.ly/Sgu84d [Accessed 16th November 2016].

Health and Safety Executive, 2015b. Personal Protective Equipment [online]. Health and Safety Executive. Available from: http://bit.ly/1p7Zelg [Accessed 4th November 2015].

Health and Safety Executive, 2015c. Construction Industry [online]. Health and Safety Executive. Available from: http://bit.ly/195hn7h [Accessed 13th November 2015].

Health and Safety Executive, 2015d. Is health surveillance required in my workplace? [online]. Health and Safety Executive. Available from: http://bit.ly/1QDzp50 [Accessed 8th March 2016] 
Health and Safety Executive, 2016. Construction health risks: Key points [online]. Health and Safety Executive. Available from: http://bit.Iy/2eYAGqn [Accessed 11 ${ }^{\text {th }}$ November 2016]

Healey, B.J. and Walker, K.T., 2009. Introduction to Occupational Health in Public Health Practice. San Francisco: Jossey-Bass.

Herman, E.S. and Chomsky, N., 1988. Manufacturing Consent - the Political Economy of the Mass Media. London: Vintage.

Kim, T.J. and von dem Knesebeck, O., 2015. Is an insecure job better for health than having no job at all? A systematic review of studies investigating the health-related risks of both job insecurity and unemployment, BMC Public Health, 15, 985.

Lincoln, Y.S. and Guba, E.G., 1985. Naturalistic Inquiry. London: Sage Publications Limited.

Marmot, M., 2004. Status Syndrome. London: Bloomsbury Publishing plc.

Marmot, M., Atkinson, T., Bell, J., Black, C., Broadfoot, P., Cumberlege, J., Diamond, I., Gilmore, I., Ham, C., Meacher, M. and Mulgan, G., 2010. Fair Society Healthy Lives. London: The Marmot Review.

McLuhan, M. and Fiore, Q., 1967. The Medium is the Massage. London: Penguin Books.

Minchin, R., Jr., Glagola, C., Guo, K., and Languell, J., 2006. Case for Drug Testing of Construction Workers. Journal of Management in Engineering. 22(1), 43-50.

Ness, K., 2010. The discourse of 'Respect for People' in UK construction. Construction Management and Economics. 28 (5) 481-93.

Oude Hengel, K.M., Blatter, B.M., Joling, C.I., van der Beek, A.J. and Bongers, P.M., 2012. Effectiveness of an intervention at construction worksites on work engagement, social support, physical workload, and need for recovery: results from a cluster randomised controlled trial. BMC Public Health, 12, 1008. 
Papadopoulos, G., Georgiadou, P., Papazoglou, C. and Michaliou K., 2010. Occupational and public health and safety in a changing work environment: An integrated approach for risk assessment and prevention. Safety Science. 48 (8), 943-949.

Payne, G. and Payne, J., 2004. Key Concepts in Social Research. London: Sage Publications Limited.

Rawlinson, F. and Farrell, P., 2010. UK construction industry site health and safety management: An examination of promotional web material as an indicator of current direction. Construction Innovation. 10 (4), 435-466.

Scott, J., 1990. A Matter of Record - Documentary Sources in Social Research. Cambridge: Polity Press.

Sherratt, F., 2015a. Legitimising Public Health Control on Sites? A critical discourse analysis of the Responsibility Deal Construction Pledge. Construction Management and Economics. 33(5-6), 444-452.

Sherratt, F., 2015b. Legitimising public health control on sites: Evaluating the UK construction industry response. In: M. Behm and C. McAleenan (Eds) Proceedings of the ClB W099 Annual Conference, 2015, 10-11 September 2015, University of Ulster, UK.

Sherratt, F., 2016. Unpacking Construction Site Safety. Chichester: Wiley-Blackwell.

Skan, D. 2015. Recognising health hazards in construction. In: C. McAleenan and D. Oloke (Eds) ICE Manual of Health and Safety in Construction, 2nd Edition, Institution of Civil Engineers. 109117.

Thompson, M. and Ellis, N., 2011. Research to explore how medium sized organisations understand occupational health issues and manage health risks, Health and Safety Executive, RR841, Norwich: HSE Books.

Tyers, C. and Hicks, B., 2012. Occupational health provision on the Olympic Park and athletes' village: Final Report, Health and Safety Executive, RR921, Norwich: HSE Books. 
Van Dijk, T. A., 2008. Discourse and Power. Basingstoke: Palgrave Macmillan.

Viester, L., Verhagen, E.A.L.M, Proper, K.I., van Dongen, J.M., Bongers, P.M. and van der Beek, A.J., 2012. VIP in construction: systematic development and evaluation of a multifaceted health programme aiming to improve physical activity levels and dietary patterns among construction workers. BMC Public Health, 12, 89.

Waite, L., 2012. In Practice London 2012, Perspectives in Public Health, 132(2) 56.

Webb, E. J., Campbell, D. T., Schwartz, R. D, and Sechrest, L., 1966. Unobtrusive Nonreactive Measures in the Social Sciences. Chicago: Rand McNally.

Wilkinson, R. and Marmot, M., 2003. The Social Determinants of Health - The Solid Facts. 2nd Edition, Copenhagen: World Health Organisation. 